livraisons

d'Histoire

de l'Architecture

\section{Livraisons de l'histoire de l'architecture}

15 | 2008

Chambres de commerces

\title{
Otello Zavaroni : la chambre de commerce et d'industrie du Havre, 1947-1957, une variante dans la reconstruction « Perret »
}

Otello Zavaroni: the Chamber of Commerce and Industry in Le Havre (1947-1957) as a variant within Perret's Reconstruction

Otello Zavaroni : die Industrie- und Handelskammer in Le Havre (1947-1957), eine Variante in Perrets Wiederaufbau

\section{Raphaëlle Saint-Pierre}

\section{OpenEdition}

Journals

Édition électronique

URL : http://journals.openedition.org/lha/169

DOI : 10.4000/lha.169

ISSN : 1960-5994

Éditeur

Association Livraisons d'histoire de l'architecture - LHA

Édition imprimée

Date de publication : 10 juin 2008

Pagination : 89-100

ISSN : 1627-4970

Référence électronique

Raphaëlle Saint-Pierre, «Otello Zavaroni : la chambre de commerce et d'industrie du Havre,

1947-1957, une variante dans la reconstruction « Perret » », Livraisons de l'histoire de l'architecture [En

ligne], 15 | 2008, mis en ligne le 10 juin 2010, consulté le 01 mai 2019. URL : http://

journals.openedition.org//ha/169; DOI : 10.4000/lha.169

Ce document a été généré automatiquement le 1 mai 2019.

Tous droits réservés à l'Association LHA 


\title{
Otello Zavaroni : la chambre de commerce et d'industrie du Havre, 1947-1957, une variante dans la reconstruction « Perret »
}

\author{
Otello Zavaroni: the Chamber of Commerce and Industry in Le Havre (1947-1957) \\ as a variant within Perret's Reconstruction \\ Otello Zavaroni : die Industrie- und Handelskammer in Le Havre (1947-1957), \\ eine Variante in Perrets Wiederaufbau
}

Raphaëlle Saint-Pierre

1 La figure et l'œuvre d'Auguste Perret ont fait l'objet de nombreux travaux depuis le début des années 1980 en France, en Allemagne comme dans les pays anglo-saxons. Ces recherches ont largement contribué à la redécouverte de l'un des pères fondateurs de l'architecture moderne ${ }^{1}$ et de son chef-d'œuvre longtemps occulté: Le Havre. Des premières études à l'inscription en $2005 \mathrm{du}$ centre reconstruit du Havre sur la liste du patrimoine mondial de l'UNESCO, la reconnaissance de ce jalon majeur de la Reconstruction en France est aujourd'hui acquise. Cependant, il semble que les bâtiments réalisés au Havre en dehors de l'atelier Perret restent encore méconnus et cette remarque s'applique notamment à la chambre de commerce dont la reconstruction devait incarner la renaissance économique de la cité au lendemain de la seconde guerre mondiale. Quelle importance revêt la construction d'un nouveau palais consulaire dans une ville où l'essentiel de l'activité est centré sur la mer et l'économie qui en dépend : l'importation et l'exportation de marchandises, la pêche, le transport et l'industrie?

\section{La reconstruction du Havre par Auguste Perret}

2 En septembre 1944, les bombardements alliés entraînent la mort de cinq mille Havrais, détruisent douze mille cinq cents immeubles et jettent à la rue quatre-vingt mille 
personnes. Dès le printemps 1945, le ministre de la Reconstruction, Raoul Dautry, confie cette formidable «table rase » à l'un des pionniers de l'architecture moderne, Auguste Perret, alors âgé de soixante et onze ans. La construction d'une ville entièrement en béton armé constitue pour Perret l'aboutissement de nombreuses années d'expérimentation de ce matériau. Autour de lui, pas moins de dix-huit architectes (Pierre-Édouard Lambert, André Le Donné, Jacques Poirrier, Jacques Tournant, etc.), pour la plupart ses élèves, forment l'atelier de reconstruction du Havre. S'y joignent des architectes havrais, ainsi que des grands noms de l'architecture, français et étrangers.

3 Au Havre, Auguste Perret applique à une vaste échelle sa doctrine du classicisme structure $l^{2}$ qui établit une correspondance étroite entre la structure en béton armé et le langage classique. Cette esthétique affirme l'ossature comme fondement de l'expression architecturale. Ses racines remontent aux rationalistes, tels Jean-Nicolas-Louis Durand ou Eugène Viollet-le-Duc, doublé d'un vocabulaire architectural fondé sur l'exploitation rationnelle des potentialités du béton armé : structure apparente, parement en béton et toit-terrasse. Cette homogénéité théorique se révèlera essentielle dans renaissance économique de la ville ${ }^{3}$.

4 Le plan d'urbanisme adopté rassemble les différentes propositions issues d'un concours interne de l'équipe. Aération et ensoleillement sont les maîtres mots des nouveaux immeubles. Toutes les dimensions des bâtiments comme celles des îlots suivent une trame orthogonale unique de six mètres vingt-quatre. En respectant en partie les anciens tracés, l'architecte et son atelier ont su éviter l'éclatement du tissu urbain et rééquilibrer la densité de la population en alternant tours et barres. La composition est marquée par un classicisme hautement affirmé : ampleur des espaces, emphase des axes qui relient places et monuments, ordonnance théâtrale des édifices publics. Le raffinement de l'architecture de Perret se lit jusque dans la légèreté des colonnades et la subtilité des teintes du béton, blanc, jaune et rose.

\section{Les étapes du chantier}

5 La chambre de commerce et d'industrie demeure intimement liée à l'histoire commerciale du Havre, port et lieu d'échanges de marchandises mais également place financière de première importance pendant près d'un siècle, précisément jusqu'en 1939. La Bourse, dont l'imposant palais est construit en 1880 par Louis Lemaître, jouait avantguerre un rôle international dans l'établissement des prix du coton, du café, des épices et des bois exotiques. Après les destructions de la guerre, c'est l'ensemble du secteur de la Bourse du Havre qu'il faut désormais entièrement repenser ${ }^{4}$. L'une des premières constructions provisoires est précisément celle de la bourse du commerce ${ }^{5}$, même si dans le procès-verbal de la réunion du 12 septembre 1947 tenue entre Barhmann, l'urbaniste du ministère de la reconstruction et de l'urbanisme responsable de la région havraise, et des collaborateurs d'Auguste Perret, il est spécifié : «L'axe de la Bourse doit rester tout à fait secondaire et ne pas être accusé par des bâtiments trop hauts pour ne pas nuire à l'ensemble de la place de l'hôtel de ville ${ }^{6}$.»

6 Dès 1947, la chambre de commerce du Havre appelle en concours tous les architectes établis dans la ville ${ }^{7}$. Les projets sont soumis à un jury réunissant autour de Meunier, président de la chambre de commerce, de Voisin, maire du Havre, et d'Auguste Perret, plusieurs architectes et membres de la chambre de commerce. Le projet des Havrais Jean 
Louvet et René Déchenaud, dont l'architecture suit les directives de Perret et emploie son vocabulaire du classicisme structurel, est retenu dans un premier temps. Peu après, l'architecte parisien et directeur d'atelier à l'École des beaux-arts, Otello Zavaroni, un esthète passionné par l'Antiquité, est nommé architecte en chef du bâtiment. Jean Louvet et René Déchenaud en deviennent les architectes d'opération. Zavaroni est l'auteur, entre autres, de la reconstruction de l'église Saint-Martin (Foucarmont, Haute-Normandie, 1959-1961) ou du foyer des retraités Louise-Voelckel (Bonneuil-sur-Marne, 1977). Zavaroni est assisté par un ingénieur-conseil en béton armé, M. Barets. L'entreprisepilote du gros œuvre et des fondations est le groupe havrais Thireau-Morel, très impliqué dans la reconstruction de la ville. La période de conception s'étend de 1947 à 1953. En mai 1953, un dessin de Zavaroni représentant la future Bourse est présenté à la foire exposition du Havre, sur le stand de la chambre de commerce et le chantier reçoit la visite de nombreux techniciens français. Son déroulement est pourtant ponctué de plusieurs conflits survenus entre le président de la chambre de commerce et Zavaroni. Le président exprime tout d'abord sa peur devant les balcons asymétriques de la façade sud mais grâce à la ténacité de l'architecte, ils ne seront pas modifiés. En revanche, le litige survenu à propos de l'entrée d'honneur au sud, face au bassin, a des répercussions sur l'allure du bâtiment. Le président estimant ses dimensions trop grandes, il décide d'interrompre les travaux pour obliger Zavaroni à dessiner une nouvelle entrée, plus discrète et sans rampe d'accès. Le chantier s'étend de 1953 à $1957^{\circ}$ et l'inauguration a lieu le 22 juillet $1957^{9}$.

\section{Le bassin du commerce}

7 Située sur l'îlot V32, place Jules Ferry et quai Georges V, la Bourse est dressée un peu plus au sud que la précédente. Elle domine majestueusement le plan d'eau du bassin du commerce qui, en plein centre de la ville, symbolise la vocation maritime du Havre. En face d'elle, s'élance la passerelle construite à la fin des années 1960, recréant ainsi l'ensemble urbain détruit. Uniquement réservée aux piétons, cette passerelle relie le quartier d'affaires à l'ancien quartier des marins. C'est un axe essentiel, tant pour les résidents du quartier un peu isolé de Saint-François qui veulent rejoindre le centre-ville, que pour les travailleurs du port autonome en relation avec la bourse-chambre de commerce. En 1945, puis en 1950, des habitants et des commerçants réclament le comblement du bassin du commerce, l'un des plus anciens du Havre (il date du début du $\mathrm{XIX}^{\mathrm{e}}$ siècle), qui appartient au port autonome du Havre, afin de rétablir une liaison entre les différents quartiers de la ville qu'il sépare. Il est cependant conservé afin de maintenir l'identité maritime de la cité havraise. L'ancien pont, qui avait survécu aux bombardements mais qui était devenu dangereux, est détruit en 1963. La municipalité désire accentuer l'image dynamique nouvelle du bassin, réservé à la navigation de plaisance. L'architecte choisi, Guillaume Gillet, souligne le caractère maritime du site en évoquant avec le haut mât de la passerelle, les voiliers et les vaisseaux qui y mouillaient autrefois. La volonté de donner à la passerelle un aspect architectural, sinon sculptural, adapté au site préside à sa conception. Elle scande avec légèreté la vaste perspective du bassin du commerce. Sa blancheur et son dynamisme asymétrique s'accordent parfaitement avec la maison de la culture dessinée par l'architecte brésilien d'Oscar Niemeyer. Tout le complexe urbanisé ${ }^{10}$ - immeubles d'habitation et commerces - qui jouxte la chambre de commerce est également conçu par l'équipe d'Otello Zavaroni 
(Raymond Audigier, Jean Louvet, René Déchenaud, Leroy, A. Rémy, Pierre Groéné) : il comprend les îlots V31b, V32a, V32b et V34.

\section{Luxe et harmonie}

8 Otello Zavaroni transforme sensiblement le premier projet en le simplifiant. Les proportions, les volumes et le parti sont à peu près les mêmes, mais l'esthétique est différente avec une nette atténuation du néoclassicisme. Sans se démarquer complètement du style Perret grâce à sa colonnade, la chambre de commerce du Havre est plus proche de l'esprit des années 1950 avec ses claustras plus modernistes que ceux de Perret ou son brise-soleil latéral. Joseph Abram analyse ainsi la différence de relecture du classicisme proposée par l'équipe d'Auguste Perret d'une part et celle d'Otello Zavaroni d'autre part :

Mais alors que l'école Perret cherche à approfondir l'approche du langage classique par un travail sur le détail et l'ornement, les architectes du palais de la Bourse préfèrent s'en tenir à une géométrisation stricte des éléments, sans allusion décorative. Ils débouchent ainsi sur une monumentalité efficace, rationnelle mais rhétorique ${ }^{11}$.

9 Étendue sur quatre-vingt-cinq mètres de long et trente-cinq mètres de large, la chambre de commerce est constituée d'une trame en béton armé régulière. Seize piliers colossaux formant péristyle sur les façades avant et arrière portent la toiture-terrasse et définissent l'ordre primaire (ill. 1).

III. 1 : La façade de la Chambre de Commerce et d'Industrie du Havre sur le Bassin du Commerce, (septembre 2007)

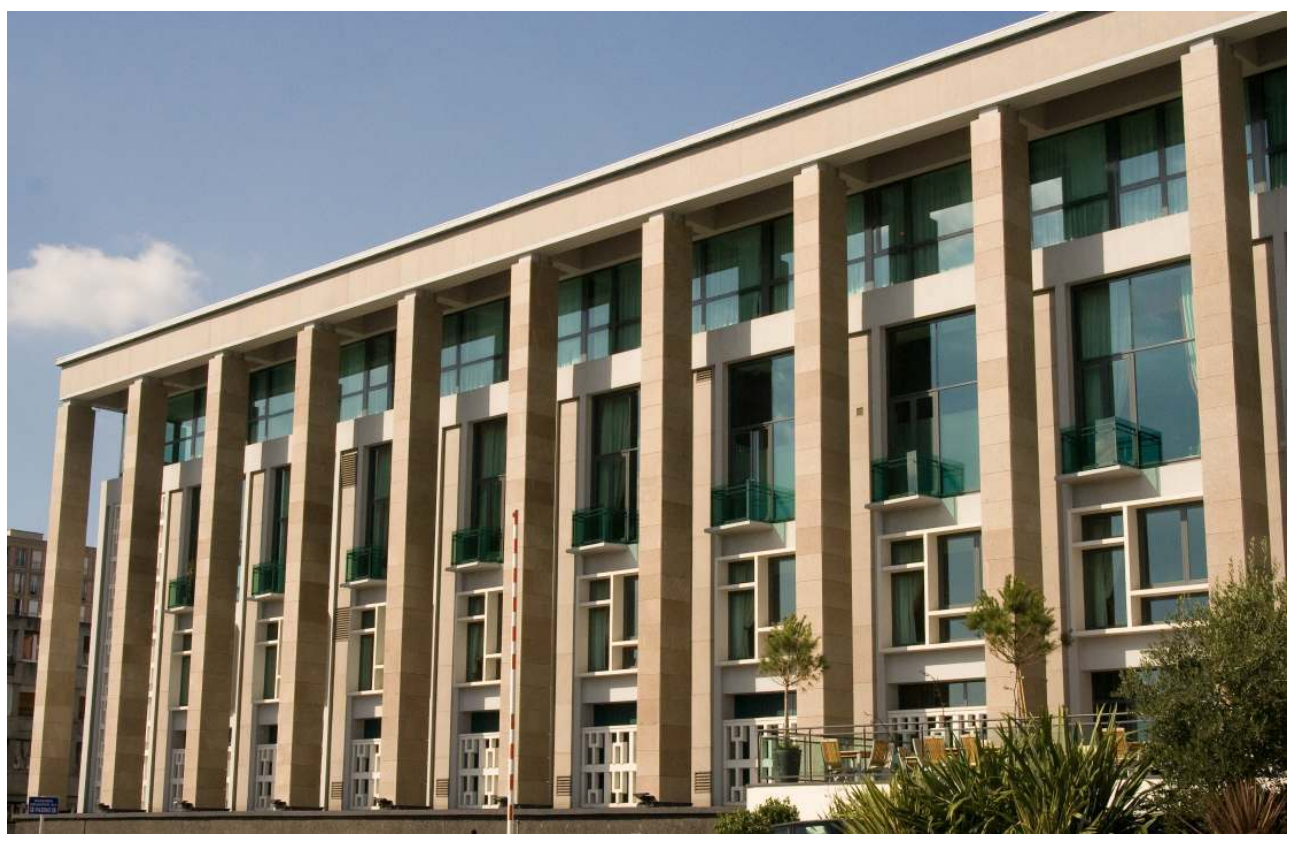

Cl. Raphaëlle Saint-Pierre

10 Ils enveloppent un bâtiment de deux étages reposant directement sur un socle, dont les nervures verticales définissent l'ordre secondaire. Sous la toiture, un étage vitré évoque clairement un attique. La structure du bâtiment étant dissociée de ses façades élevées en retrait, les ouvertures sont traitées avec plus de liberté. Ainsi, selon la destination des 
espaces et l'orientation, des claustras, des paralumes, des fenêtres à encadrement ou des baies vitrées animent les façades (ill. 2).

III. 2 : Les claustras de la façade sur le Bassin du Commerce, (septembre 2007)

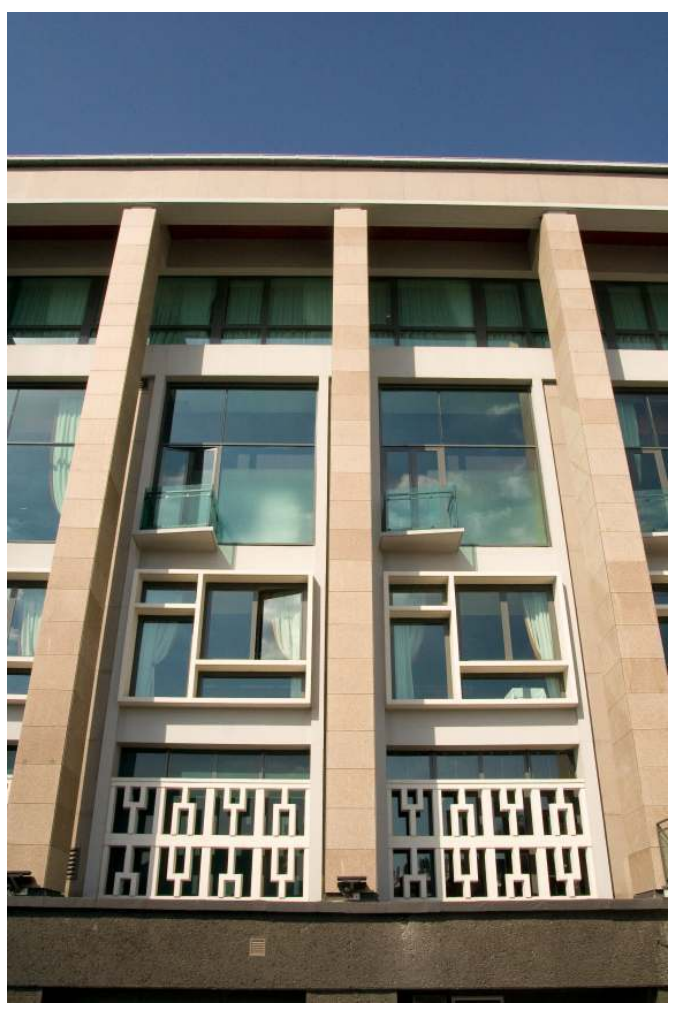

Cl. Raphaëlle Saint-Pierre

11 Un brise-soleil, des balcons en biais, et les menuiseries complètent le décor. Les piliers étaient revêtus à l'origine de plaques de granit de Suède adouci, d'un gris bleu aux reflets changeants. Deux entrées desservent le bâtiment. Du côté du bassin du commerce, quelques marches mènent à l'entrée d'honneur. Sur la place Jules Ferry ${ }^{12}$, l'entrée des usagers est précédée d'un perron monumental. Elle donne accès au vestibule d'honneur situé dans l'est du bâtiment et d'où le grand escalier monte jusqu'au deuxième étage (ill. 3). 


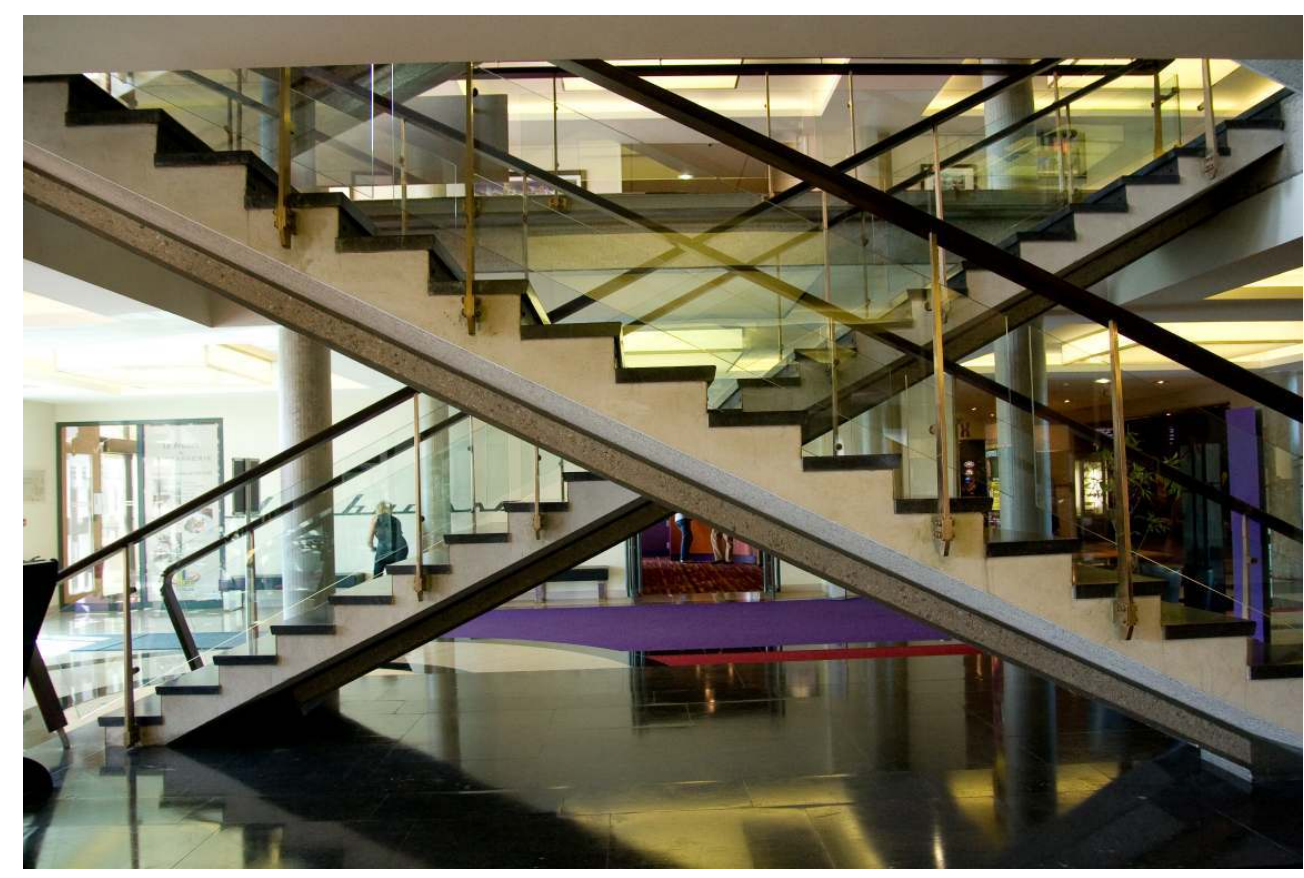

Cl. Raphaëlle Saint-Pierre

Cet escalier est composé d'un double jeu de volées en $\mathrm{X}$, aux marches de marbre noir et encadré par des balustrades de bronze portant des plaques de verre. Des escaliers secondaires ont été disposés à chaque extrémité de l'édifice ainsi que des ascenseurs.

Dans le programme, il est précisé que les installations de la chambre de commerce et celles de la Bourse doivent être nettement distinguées ${ }^{13}$. La division de ces deux éléments s'effectue par niveau : les services de la Bourse occupent tout le rez-de-chaussée surélevé et ceux de la chambre de commerce, les premier et deuxième étages. Au rez-de-chaussée, tous les services sont attenants à la salle des pas-perdus qui représente le centre même des affaires, de sorte que l'on puisse accéder aux deux salles de call, consacrées au coton et au café, ainsi qu'à la salle de lecture et de correspondance. Des cabines téléphoniques sont disposées dans l'enceinte même de la salle des pas-perdus. Les locaux de la chambre de commerce sont répartis au sud sur deux étages : bureaux du président, du secrétaire général et des vice-présidents, secrétariat, comptabilité, archives. Des logements sont prévus au troisième étage ainsi que dans une partie du soubassement. Ce bâtiment possède également de nombreux locaux d'apparat : la salle des fêtes et la bibliothèque au deuxième étage sud, un petit musée colonial au deuxième étage nord, la salle de conférences au deuxième étage angle nord-ouest, les salles de séances et de commissions. À l'intérieur, l'ossature de béton armé est conservée apparente pour les plafonds et les poteaux, bruts de décoffrage. Le sol est revêtu de dalles de comblanchien et les murs de travertin.

14 La vaste salle des pas-perdus mesure quarante mètres sur quinze et possède une hauteur sous plafond de douze mètres. Son volume correspond aux trois niveaux des étages. Elle est entièrement vitrée du côté de la façade nord - les seize baies vitrées sont constituées chacune d'une dalle de verre carrée de trois mètres quatre-vingt-dix - mais également sur ses faces intérieures puisqu'elle n'est séparée du reste du bâtiment que par des parois de glaces et des claustras de béton doré. Une fresque monumentale, longue de soixante 
mètres, est consacrée aux activités industrielles, commerciales et portuaires du Havre. Le plafond en béton précontraint à ossature apparente est soutenu par des poutres jumelées d'une portée de quatorze mètres entre lesquelles s'insèrent les dispositifs d'éclairage. Une discrète polychromie complète la décoration du bâtiment.

\section{Un ultime plaidoyer du style Beaux-Arts ?}

La nouvelle chambre de commerce du Havre fera l'objet d'un important programme de décoration faisant appel à des figures renommées de l'École des beaux-arts de Paris dans l'idée d'une véritable synthèse des arts plastiques. Dès les premiers projets, les créations de trois artistes sont intégrées à son architecture ${ }^{14}$. Nicolas Untersteller (1900-1967), membre de l'Institut, professeur de fresque à l'École des beaux-arts de Paris dont il devient le directeur de 1948 à 1967, s'est notamment illustré aux églises du Saint-Esprit (1927-1933) et de Saint-Pierre de Chaillot (1933-1938) à Paris mais également par des vitraux au ciment à l'église Sainte-Thérèse de Metz (1937-1954) - chef-d'œuvre en béton armé conçu par l'architecte Roger-Henri Expert (1882-1955). Dans la salle des pas-perdus de la Bourse, il réalise une fresque très colorée, visible depuis l'extérieur du bâtiment qui symbolise l'activité du port du Havre: la navigation, les industries, l'importation de produits exotiques (ill. 4).

III. 4 : Fresque de Nicolas Untersteller, salle des Pas Perdus de la Bourse, (septembre 2007)

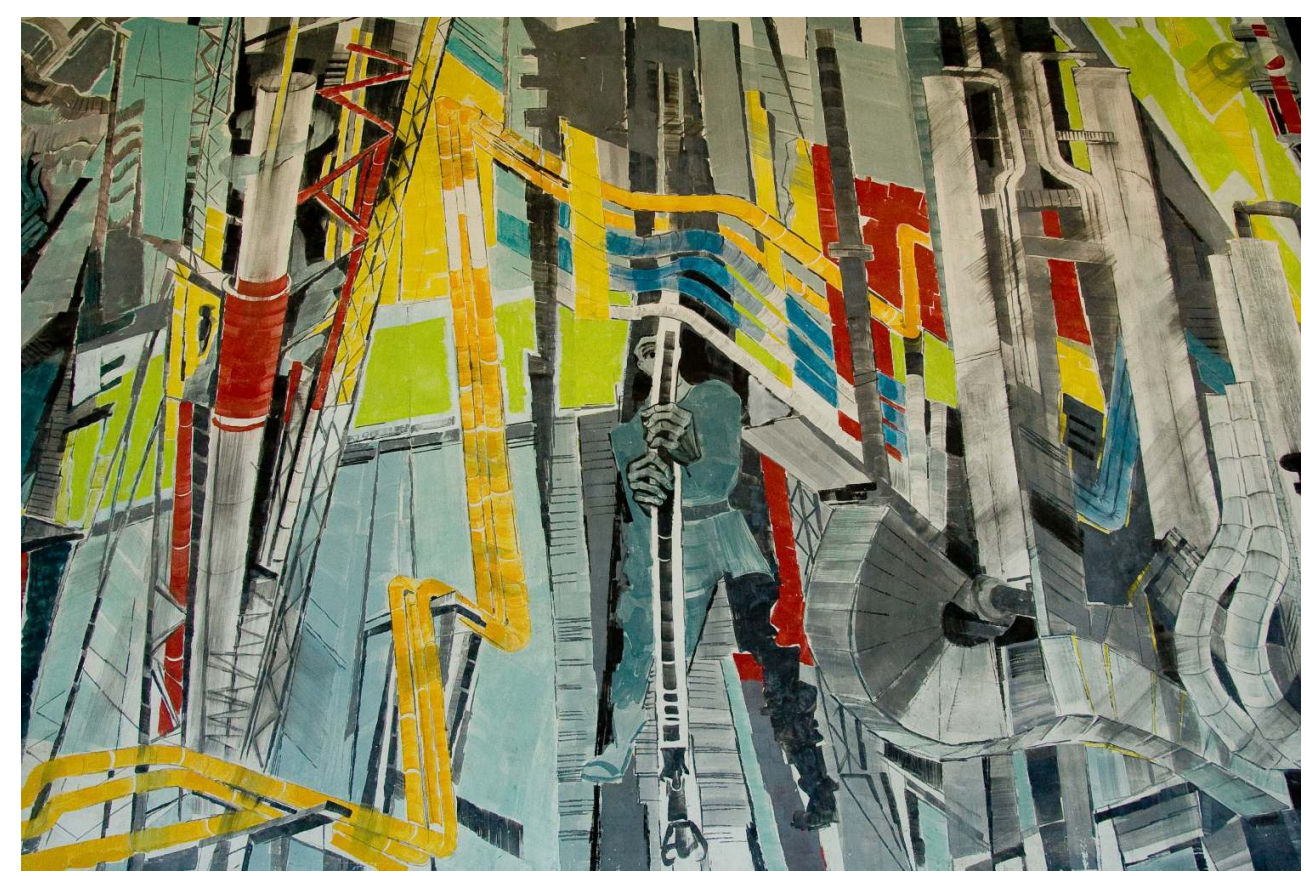

Cl. Raphaëlle Saint-Pierre

16 À huit mètres au-dessus du sol, elle s'étend sur trente-deux mètres de large pour quatre mètres de haut. Henri Lagriffoul (1907-1981), grand prix de Rome, est l'auteur des sculptures en haut-relief en travertin du petit hall d'entrée ouest. Elles ont pour thème les activités navales et portuaires comme la construction des bateaux, la pêche ou les périls de la mer (aujourd'hui déplacées). Paul Lemagny, membre de l'Institut et Grand Prix de Rome, dessine pour la salle des fêtes une immense fresque sur stuc de quarante- 
cinq mètres traitant de la vie de la faune marine qui s'étend sur toute la longueur du mur face au bassin du commerce (ill. 5).

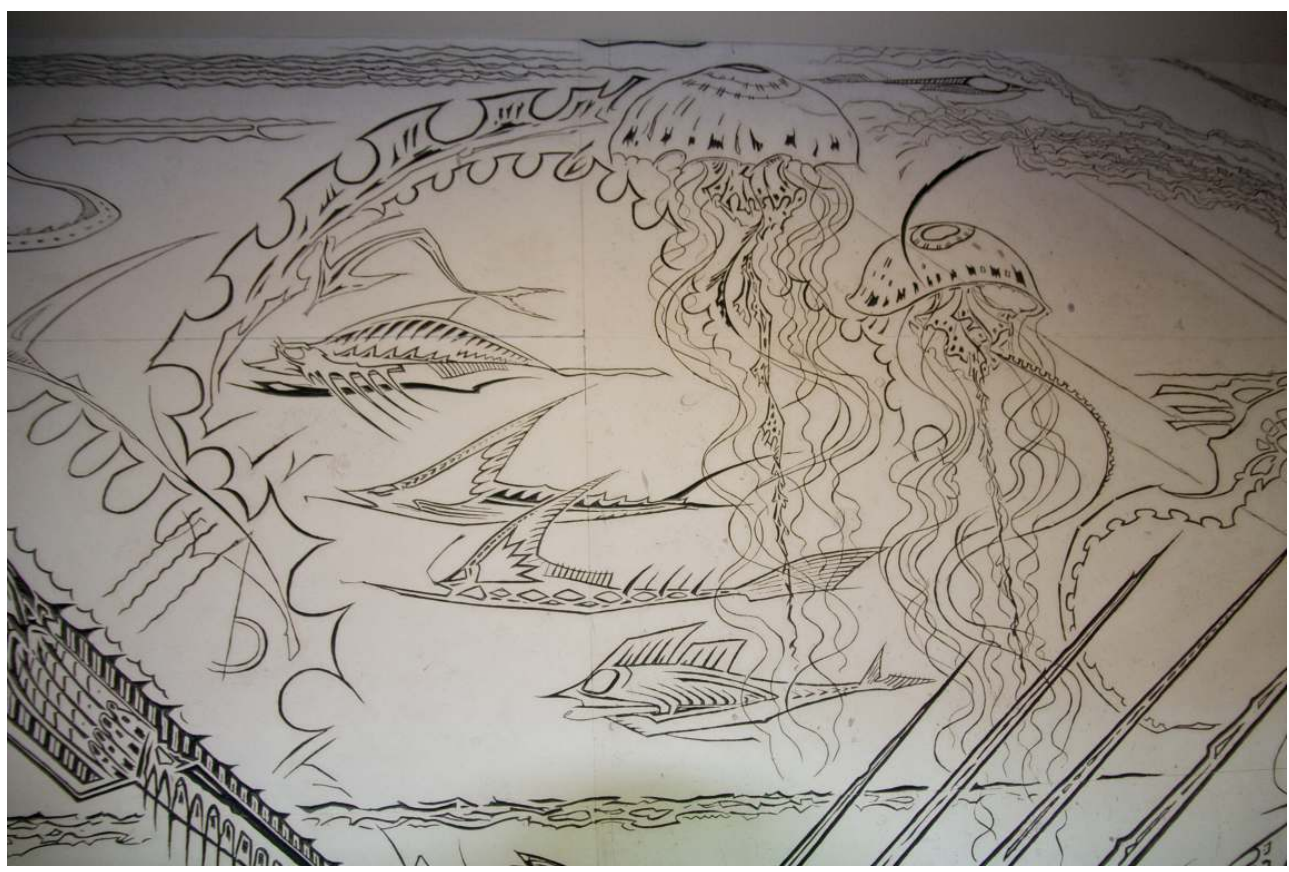

Cl. Raphaëlle Saint-Pierre

\begin{abstract}
un fond blanc). Elle représente un paysage sous-marin fantastique peuplé de sirènes et de monstres dont les lignes dynamiques sont caractéristiques des illustrations et du graphisme des années 1950.
\end{abstract} acajou du bureau du président est complétée par le mobilier de Jean Royère ${ }^{15}$ : fauteuils, tables, lustre et canapé. Un panneau de laque noir et or figurant un port entouré d'une multitude de bateaux, trône au-dessus du canapé. C'est une œuvre de Bernard Dunand ${ }^{16}$, fils du célèbre décorateur et créateur de laques Jean Dunand. En 1968, des chaises de Royère avec ornements en bronze doré sont ajoutées, auxquelles s'ajoutent en 1971, une nouvelle commande de huit lustres en fer forgé doré et leurs cent soixante abat-jour correspondants. Spécialiste des programmes luxueux de cette envergure, Jean Royère, l'un des décorateurs les plus célèbres des années $1950^{17}$, s'appliquera à dessiner pour la Chambre de Commerce du Havre, un mobilier Consulat afin de rappeler la création de la Bourse en 1802.

Le bâtiment est protégé depuis 1995 dans le cadre d'une Z.P.P.A.U.P. (zone de protection du patrimoine architectural urbain et paysager). Afin de s'agrandir, les services de la chambre de commerce et d'industrie ont intégré un nouvel édifice situé sur le bassin Vauban. La Bourse a, quant à elle, été rachetée en 2005, l'année de l'inscription du centreville reconstruit du Havre au patrimoine mondial, par le groupe Partouche pour y implanter un ensemble comportant un casino, un hôtel et un restaurant. Une concertation avec les nouveaux propriétaires a alors été engagée par la Ville et Docomomo France pour la bonne conservation de l'architecture et des éléments de 
décoration, notamment les fresques. Parmi les modifications notables, on remarque en particulier le doublement de la salle des pas-perdus par la création d'un plancher à mihauteur et la pose d'un faux-plafond ainsi que le cloisonnement de la salle des fêtes. Cette nouvelle destination rejoint un ancien projet d'aménagement de la place Gambetta, située à l'extrémité du bassin du commerce. En effet, en 1966-1967, Guillaume Gillet avait proposé d'y construire des commerces, un parking souterrain ainsi qu'un bâtiment circulaire accueillant tout à la fois un théâtre, un centre culturel, un casino municipal et un bowling. Mais seuls un théâtre et une maison de la culture y seront construits par Oscar Niemeyer entre 1978 et 1982.

L'inscription du centre reconstruit du Havre sur la liste du patrimoine mondial de l'UNESCO, en juillet 2005 constitue un premier pas vers la reconnaissance de l'architecture française des années 1950, pourtant riche en innovations mais encore largement décriée par les contemporains. L'intérêt patrimonial du centre-ville du Havre réside bien sûr dans son intérêt historique mais également esthétique et technique. Il répond ainsi aux trois critères édictés par l'Unesco: un «ensemble architectural illustrant une période significative de l'histoire humaine », un « témoignage d'un échange d'influences considérable pendant une période donnée ou dans une aire culturelle déterminée, sur le développement de l'architecture ou de la technologie, des arts monumentaux, de la planification des villes ou de la création de paysages » et enfin considérer cet ensemble comme un " chef-d'œuvre du génie créateur humain ». Le Havre rejoint ainsi Brasilia d'Oscar Niemeyer et de Lucio Costa, inscrite au Patrimoine Mondial dès 1987 et attend la désignation prochaine de Chandigarh de Le Corbusier. Alors que les voyageurs ne découvrent plus la France depuis Le Havre, ce classement devrait amener de nouveaux flux touristiques vers une cité longtemps mal-aimée, tandis que les Havrais, encore partagés entre rejet et fierté, commencent à se réapproprier le chef d'œuvre de Perret.

\section{NOTES}

1. 1. Voir en particulier Joseph Abram, Jean-Louis Cohen et Guy Lambert, Encyclopédie Perret, Paris, Monum/Le Moniteur/IFA, 2002, 446 p., Perret et la poétique du béton, exposition de l'IFA et de la ville du Havre, 2002, Joseph Abram, Guy Lambert, Christophe Laurent, Auguste Perret: Anthologie des écrits, conférences et entretiens, Paris, Le Moniteur, 2006, 480 p.

2. 2. Joseph Abram, Perret et l'école du classicisme structurel, EAN/SRA, 1985, 2 t., 315-136 p. ; L'Équipe Perret au Havre. Utopie et compromis d'une reconstruction, Paris, Bureau de la recherche architecturale, 1989, $268 \mathrm{p}$.

3. 3. Joseph Abram, Sylvie Barot, Élizabeth Chauvin, Les Bâtisseurs, l'album de la reconstruction du Havre, Le Havre, Éditions Point de vues, musée Malraux, 2002, 132 p.

4. 4. «L'ancienne Bourse sera rasée », Le Havre Libre, 5 juin 1947, p. 1 ; « La reconstruction du palais de la bourse ", La Revue de la Porte Océane ${ }^{\circ}{ }^{27}$, juillet 1947 ; "Fin de la destruction ", Le Havre Libre, $1^{\mathrm{er}}$ juillet 1949, p. 3.

5. 5. « La bourse de commerce provisoire », Le Havre Libre, 30 décembre 1944, p. 1. 
6. 6. Arch. mun. de la ville du Havre; fonds contemporain, demande de permis de construire PC 201/1953, PC 555/55 ; dossier documentaire 30/6.1.

7. 7. Arch. de la C.C.I. du Havre, 1997-3-3.

8. 8. Archives de la chambre de commerce et d'industrie du Havre, 1997-3-5.

9. 9. « La Bourse sort de terre », Le Havre Libre, 11 février 1955, p. 4 ; « La nouvelle Bourse ", Escale, revue du personnel du port autonome du Havre n ${ }^{\circ} 36$, mars 1956, p. 8 ; « Voici le vrai et définitif visage du quartier de la Bourse », Le Havre, 7 juin 1956; «La bourse de commerce serait inaugurée l'automne prochain », Le Havre Libre, 21 mai 1957, p. 3.

10. 10. "Remembrement et reconstruction des immeubles entourant la Bourse ", Le Havre Libre, 6 décembre 1954, p. 3.

11. 11. Joseph Abram, Le Havre, la ville reconstruite par Auguste Perret, dossier UNESCO, 5 août 2003.

12. 12. « Le jardin de la Bourse », Le Havre, 17 juin 1959 ; « La place de la Bourse », Le Havre , 23 juillet 1959.

13. 13. Archives de la chambre de commerce et d'industrie du Havre, 1997-3-6.

14. 14. "Les travaux de décoration de la Bourse avancent rapidement ", Le Havre, 20-21 juillet 1957.

15. 15. Archives du musée des arts décoratifs, fonds Jean Royère R.1650.

16. 16. Archives de la chambre de commerce et d'industrie du Havre, 1997-3-7.

17. 17. Pierre-Emmanuel Martin-Vivier, Jean Royère, Paris, Norma, 2001, 320 p.

\section{RÉSUMÉS}

La chambre de commerce et d'industrie du Havre est achevée en 1957. Elle succède à la bourse, détruite par les bombardements de septembre 1944, avec une grande partie de la ville. Élevé à l'intérieur du périmètre du centre reconstruit, qui a été inscrit en 2005 sur la liste du patrimoine mondial de l'UNESCO, ce bâtiment monumental dresse son ordonnance majestueuse face au bassin du Commerce. L'architecte, Otello Zavaroni, professeur à l'École des beaux-arts de Paris, n'appartient pas à l'atelier de reconstruction de la ville, piloté par Auguste Perret et ses disciples. Bien qu'elle manifeste une réappropriation du langage classique, la conception de la chambre de commerce et d'industrie se distingue nettement du classicisme structurel de Perret déployé dans l'ensemble du centre-ville. L'intervention de décorateurs, à l'image de celle du fresquiste Nicolas Untersteller, et du décorateur Jean Royère, s'accorde avec l'atmosphère luxueuse du lieu. Sa reconversion récente en casino soulève la question de la protection de ce type d'édifices des années 1950, devenus difficiles à utiliser dans leur destination originelle.

The Chamber of Commerce and Industry in Le Havre was achieved in 1957. It took the place of the Stock Exchange destroyed by September 1944 bombardment along with a big part of the city. It is located within the rebuilt centre which was listed as a World Heritage site in 2005. This monumental building made of reinforced concrete, with colossal pillars, stands in front of the dock of Commerce. The architect Otello Zavaroni was also a teacher at the Ecole des Beaux-Arts in Paris. But he was not a member of the Reconstruction workshop of the city ran by Auguste Perret and his disciples. Though the architecture of the Chamber of Commerce shows Classical 
language, it is more geometric than the Structural Classicism of the Le Havre buildings by Perret. The collaboration with artists such as the fresco painter Nicolas Untersteller and the decorator Jean Royère matches the luxurious atmosphere of this place. The building has been converted into a casino lately: this raises the issue of the preservation of this kind of 1950 buildings whose original purpose is hard to maintain now.

Der Bau der Industrie- und Handelskammer von Le Havre wurde 1957 beendet. Das neue Gebäude trat an die Stelle der Börse, die im September 1944 mit einem weitgehenden Teil der Stadt zerbombt wurde. In dem Areal der wiederaufgebauten Stadtmitte errichtet, welche im Jahr 2005 von der UNESCO in die Liste des Welterbes aufgenommen wurde, erhebt sich dieses prächtige, monumental gegliederte Gebäude gegenüber dem Bassin du Commerce (Hafenbecken). Der Architekt Otello Zavaroni, Lehrer an der Pariser École des beaux-arts, gehörte nicht dem von Auguste Perret und seinen Schülern geleiteten Büro für Wiederaufbau der Stadt an. Obwohl die Bauart der Industrie- und Handelskammer die klassische Bausprache übernimmt, unterscheidet sie sich augenscheinlich von dem strukturellen Klassizismus, den Perret in der ganzen Stadtmitte anwendet. Die Beteiligung von Dekorateuren wie Jean Royère oder dem Freskenmaler Nicolas Untersteller unterstreicht die luxuriöse Atmosphäre des Ortes. Die neueste Umgestaltung der Handelskammer in ein Casino wirft die Frage nach dem Schutz solcher Gebäude aus den fünfziger Jahren, die in ihrer originellen Bestimmung nicht mehr verwendbar sind, auf.

\section{AUTEUR}

\section{RAPHAËLLE SAINT-PIERRE}

Raphaëlle Saint-Pierre a soutenu un DEA d'histoire de l'architecture, Les Villas des années 50 en France, à Paris I en 2002. Elle a été chargée, en 2003-2004, d'une mission avec Docomomo France sur la reconstruction du Havre en vue de son inscription sur la liste du patrimoine mondial. Elle est l'auteur d'ouvrages : Villas 50 en France (Paris, Éditions Norma, 2005), Trésors des châteaux de France (Paris, Éditions Molière, 2007) et d'articles, en particulier « Les maisons de Georges Adilon », Architectures à vivre $\mathrm{n}^{\circ} 38$, septembre 2007, "Les maisons de Claude Parent ", Architectures à vivre $n^{\circ} 31$, août 2006, «Porte Océane, Das Stadttor von Le Havre », Bauwelt $n^{\circ} 45$, novembre 2005, «Le Havre : quand le béton devient patrimoine ", L'Histoire $\mathrm{n}^{\circ} 303$, novembre 2005 ou encore « Auguste Perret au patrimoine de l'humanité », La Règle du Jeu n 28, mai 2005. Adresse électronique : raphaellesaintpierre@yahoo.fr 